\title{
Valoración analítica de opciones asiáticas aritméticas continuas mediante transformadas de Fourier
}

\author{
Analytical pricing of continuous arithmetic \\ Asian options using Fourier transforms
}

\author{
Diana L. Guavita F.* \\ John F. Moreno T.*
}

* Magíster en Finanzas. Colombian Shared Services (jefe de Costos). Bogotá, Colombia. [dguavita751@gmail.com]

** Magíster en Matemática Aplicada. Universidad Externado de Colombia. Bogotá, Colombia. [jhon.moreno@uexternado.edu.co] [ORCID: 0000-0002-2772-6931].

Artículo recibido el 15 de octubre de 2019.

Aceptado el 20 de noviembre de 2019.

Para citar este artáculo:

Guavita, D. y Moreno Trujillo, J. F. (2019). Valoración analítica de opciones asiáticas aritméticas continuas mediante transformadas de Fourier. ODEON, 17, pp. 157-170.

DOI: https://doi.org/10.18601/17941113.n17.06 


\section{Resumen}

En este artículo se presenta la deducción de la ecuación diferencial parcial de segundo orden asociada al problema de valoración de opciones asiáticas aritméticas continuas, junto con la aplicación de la transformada de Fourier sobre los términos de la ecuación resultante después de una serie de cambios de variable. Se obtiene una solución analítica para el valor de este tipo de opciones.

Palabras clave: opciones asiáticas; valoración; ecuación diferencial parcial; transformada de Fourier.

Clasificación JEL: C02, C32, G13.

\section{Abstract}

The second-order partial differential equation associated with the problem of pricing continuous arithmetic Asian options is presented, together with the application of the Fourier transform on the terms of the resulting equation after a series of variable changes. An analytical solution is obtained for the value of this type of options.

Key words: Asian options; pricing; partial differential equation; Fourier transform.

JEL classification: C02, C32, G13. 


\section{Introducción}

Los derivados financieros son un tipo particular de activo (contrato) cuyo valor depende del precio de otro activo denominado subyacente. Los tipos básicos de derivados financieros son los futuros y forwards, los swaps y las opciones. En particular, las opciones son contratos que otorgan a su poseedor el derecho, mas no la obligación, de negociar una determinada cantidad del activo subyacente en o antes de una fecha futura específica $(T)$ y por un valor determinado. Dentro de las denominadas opciones exóticas se encuentran las asiáticas, que son un tipo de opción cuyo valor al vencimiento depende del promedio del precio del activo subyacente durante la vida del contrato. Las opciones asiáticas se pueden clasificar, en función de la forma como se calcula el promedio del precio, en: opciones aritméticas y opciones geométricas. Las opciones asiáticas de tipo call dan a su poseedor el derecho a comprar el activo subyacente en una fecha futura específica y por un valor equivalente al precio promedio del activo subyacente. De forma análoga, las opciones put dan a su poseedor el derecho a vender el activo subyacente por un valor equivalente al precio promedio.

En el caso de las opciones asiáticas geométricas existe una solución analítica cerrada al problema de determinar su valor en $t \leq T$, como se expone en Barucci, Polidoro y Vespri (2001). Sin embargo, el caso de las opciones aritméticas es más complejo porque el promedio aritmético de variables lognormales no está lognormalmente distribuido y, en general, no se tiene una forma analítica para su distribución. Varios métodos se han propuesto para aproximar el valor de este tipo de opciones, por ejemplo,Yor (2001) utiliza la transformada de Laplace en la variable tiempo de la opción, aunque esta transformación solamente es aplicable en determinados casos. En Rogers y Shi (1995) el problema de valoración se transforma en la resolución de una ecuación diferencial parcial en dos variables, con el inconveniente de que no se tiene una solución analítica de esta ecuación y los métodos numéricos aplicables presentan problemas de estabilidad y convergencia. En Zhang (2003) se presenta una teoría de muestreo continuo que genera una ecuación diferencial parcial cuya solución es aproximada por el método de perturbación. En el trabajo de Vecer (2001) se presenta una aproximación al problema en la cual considera la opción asiática como análoga a una opción sobre una cuenta de trading, desarrollando una ecuación diferencial parcial unidimensional para la valoración, pero que es limitada a escenarios muy específicos. En Dubois y Lelièvre (2004) se deriva un método numérico convergente y eficiente para resolver la ecuación propuesta en Rogers y Shi. 
En este artículo se deriva la ecuación diferencial parcial (EDP) asociada al problema de la valoración de opciones asiáticas aritméticas continuas, y se presentan metodologías para la solución de esta ecuación mediante la aplicación de las transformaciones integrales de Fourier y Laplace.

\section{Derivación de la ecuación diferencial parcial de valoración para opciones asiáticas aritméticas}

Se considera un mercado financiero libre de arbitraje definido sobre un espacio de probabilidad filtrado $\left(\Omega,\left(\mathcal{F}_{t}\right)_{t \geq 0}, P\right)$, conformado por un activo libre de riesgo $\left(B_{t}\right)$, asociado a una tasa de interés constante y conocida $r_{f}$ y que satisface la ecuación diferencial:

$$
d B_{t}=r_{f} B_{t} d t
$$

o de forma equivalente,

$$
B_{t}=B_{0} e^{r_{f} t}
$$

y un activo riesgoso $\left(S_{t}\right)$ que satisface la ecuación diferencial estocástica:

$$
d S_{t}=\mu S_{t} d t+\sigma S_{t} d W_{t}
$$

donde $\mu$ y $\sigma$ son constantes que representan la tasa instantánea de rentabilidad $\mathrm{y}$ volatilidad del activo respectivamente, y $W_{t}$ es un movimiento Browniano estándar. Mediante la aplicación del lema de Itô se verifica que:

$$
S_{t}=S_{0} e^{\left(\mu-\frac{\sigma^{2}}{2}\right) t+\sigma W_{t}}
$$

Adicionalmente, se considera una opción asiática aritmética continua con tasa promedio definida por la suma acumulada del precio del activo subyacente,

$$
A_{t}=\int_{0}^{t} S_{u} d u
$$

o en notación diferencial,

$$
d A_{t}=S_{t} d t
$$

Considerando que la opción asiática tiene función de pago final $\phi\left(S_{T}, \frac{A_{T}}{T}\right)$ en la fecha de maduración $T$, el teorema fundamental de valoración de activos 
contingentes establece que el valor de la opción en cualquier instante $t \leq T$ está dado por:

$$
V\left(t, S_{t}, A_{t}\right)=e^{-r_{f}(T-t)} E^{Q}\left[\phi\left(S_{T}, \frac{A_{T}}{T}\right) \mid \mathcal{F}_{t}\right]
$$

donde $E^{Q}\left[\cdot \mid \mathcal{F}_{t}\right]$ denota el valor esperado bajo una medida de probabilidad riesgo neutral $Q$ condicional a la información disponible hasta el instante $t$. Aplicando el lema de Itô multidimensional se tiene que el diferencial de $V\left(t, S_{t}, A_{t}\right)$ es:

$$
\begin{aligned}
d V & =\frac{\partial V}{\partial t} d t+\frac{\partial V}{\partial S_{t}} d S_{t}+\frac{\partial V}{\partial A_{t}} d A_{t}+\frac{1}{2}\left[\frac{\partial^{2} V}{\partial S_{t}^{2}}\left(d S_{t}\right)^{2}+\frac{\partial^{2} V}{\partial A_{t}^{2}}\left(d A_{t}\right)^{2}+2 \frac{\partial^{2} V}{\partial S_{t} \partial A_{t}}\left(d S_{t}\right)\left(d A_{t}\right)\right] \\
& =\frac{\partial V}{\partial t} d t+\frac{\partial V}{\partial S_{t}}\left(\mu S_{t} d t+\sigma S_{t} d W_{t}\right)+S_{t} \frac{\partial V}{\partial A_{t}} d t+\frac{\sigma^{2} S_{t}^{2}}{2} \frac{\partial^{2} V}{\partial S_{t}^{2}} d t \\
& =\left(\frac{\partial V}{\partial t}+\mu S_{t} \frac{\partial V}{\partial S_{t}}+\frac{\sigma^{2} S_{t}^{2}}{2} \frac{\partial^{2} V}{\partial S_{t}^{2}}+S_{t} \frac{\partial V}{\partial A_{t}}\right) d t+\left(\sigma S_{t} \frac{\partial V}{\partial S_{t}}\right) d W_{t}
\end{aligned}
$$

Si se conforma un portafolio (П) con una posición larga en la opción asiática aritmética y una posición corta en $\Delta$ unidades del activo subyacente, el valor en $t$ de este portafolio está dado por:

$$
\Pi_{t}=V\left(t, S_{t}, A_{t}\right)-\Delta S_{t}
$$

y el cambio en su valor es:

$$
\begin{aligned}
d \Pi_{t} & =d V-\Delta d S_{t} \\
& =\left(\frac{\partial V}{\partial t}+\mu S_{t} \frac{\partial V}{\partial S_{t}}+\frac{\sigma^{2} S_{t}^{2}}{2} \frac{\partial^{2} V}{\partial S_{t}^{2}}+S_{t} \frac{\partial V}{\partial A_{t}}\right) d t+\left(\sigma S_{t} \frac{\partial V}{\partial S_{t}}\right) d W_{t}-\Delta\left(\mu S_{t} d t+\sigma S_{t} d W_{t}\right) \\
& =\left(\frac{\partial V}{\partial t}+\mu S_{t} \frac{\partial V}{\partial S_{t}}+\frac{\sigma^{2} S_{t}^{2}}{2} \frac{\partial^{2} V}{\partial S_{t}^{2}}+S_{t} \frac{\partial V}{\partial A_{t}}-\Delta \mu S_{t}\right) d t+\left(\sigma S_{t} \frac{\partial V}{\partial S_{t}}-\Delta \sigma S_{t}\right) d W_{t}
\end{aligned}
$$

El componente estocástico del cambio en el valor del portafolio puede ser eliminado si se selecciona $\Delta=\frac{\partial V}{\partial S_{t}}$, de forma que:

$$
d \Pi_{t}=\left(\frac{\partial V}{\partial t}+\mu S_{t} \frac{\partial V}{\partial S_{t}}+\frac{\sigma^{2} S_{t}^{2}}{2} \frac{\partial^{2} V}{\partial S_{t}^{2}}+S_{t} \frac{\partial V}{\partial A_{t}}-\frac{\partial V}{\partial S_{t}} \mu S_{t}\right) d t+\left(\sigma S_{t} \frac{\partial V}{\partial S_{t}}-\frac{\partial V}{\partial S_{t}} \sigma S_{t}\right) d W_{t}
$$


de donde se llega finalmente a que:

$$
d \Pi_{t}=\left(\frac{\partial V}{\partial t}+\frac{\sigma^{2} S_{t}^{2}}{2} \frac{\partial^{2} V}{\partial S_{t}^{2}}+S_{t} \frac{\partial V}{\partial A_{t}}\right) d t
$$

Dada la ausencia de oportunidades de arbitraje en el mercado, la expresión (9) debe ser igual al rendimiento que se tiene si se invierte el valor del portafolio a la tasa libre de riesgo $r_{f}$ durante un periodo de tiempo infinitesimal entre $t$ y $t+d t$, es decir:

$$
d \Pi_{t}=r_{f} \Pi_{t} d t=\left(r_{f} V-r_{f} \Delta S_{t}\right) d t=\left(r_{f} V-r_{f} \frac{\partial V}{\partial S_{t}} S_{t}\right) d t
$$

Igualando (9) y (10) se tiene que:

$$
\left(\frac{\partial V}{\partial t}+\frac{\sigma^{2} S_{t}^{2}}{2} \frac{\partial^{2} V}{\partial S_{t}^{2}}+S_{t} \frac{\partial V}{\partial A_{t}}\right) d t=\left(r_{f} V-r_{f} \frac{\partial V}{\partial S_{t}} S_{t}\right) d t
$$

y se llega la EDP de segundo orden asociada al problema de valoración de opciones asiáticas:

$$
\frac{\partial V}{\partial t}+\frac{\sigma^{2} S_{t}^{2}}{2} \frac{\partial^{2} V}{\partial S_{t}^{2}}+r_{f} S_{t} \frac{\partial V}{\partial S_{t}}+S_{t} \frac{\partial V}{\partial A_{t}}-r_{f} V=0
$$

junto con la condición de frontera $V\left(T, S_{T}, A_{T}\right)=\phi\left(S_{T}, \frac{A_{T}}{T}\right)$.

Existen cuatro tipos de opciones asiáticas aritméticas continuas dependiendo de la forma de la función $\phi\left(S_{T}, \frac{A_{T}}{T}\right)$, que son:

- Opción call con strike fijo: $\phi\left(S_{T}, \frac{A_{T}}{T}\right)=\max \left(\frac{A_{T}}{T}-K ; 0\right)$.

- Opción put con strike fijo: $\phi\left(S_{T}, \frac{A_{T}}{T}\right)=\max \left(K-\frac{A_{T}}{T} ; 0\right)$.

- Opción call con strike flotante: $\phi\left(S_{T}, \frac{A_{T}}{T}\right)=\max \left(S_{T}-\frac{A_{T}}{T} ; 0\right)$.

- Opción put con strike flotante: $\phi\left(S_{T}, \frac{A_{T}}{T}\right)=\max \left(\frac{A_{T}}{T}-S_{T} ; 0\right)$.

\section{Solución analítica de la EDP de valoración aplicando la transformada de Fourier}

La transformada de Fourier de una función $f(x)$ está definida como: 


$$
F[f(x)]=g(w)=\int_{-\infty}^{\infty} f(x) e^{-i w x} d x
$$

y la transformada inversa de Fourier se define como:

$$
F^{-1}[g(w)]=f(x)=\frac{1}{2 \pi} \int_{-\infty}^{\infty} g(w) e^{i w x} d w
$$

El objetivo de aplicar esta transformación es cambiar el espacio sobre el cual está definida una función o ecuación de interés, por otro en el cual esta función o ecuación sean más sencillas de tratar. Algunas de las propiedades de esta transformación son:

1.

$$
F[\alpha f(x)+\beta g(x)]=\alpha F[f(x)]+\beta F[g(x)]
$$

2.

$$
F\left[\frac{\partial^{n} f}{\partial x^{n}}\right]=(i w)^{n} g(w)
$$

3.

$$
F\left[e^{i \alpha x} f(x)\right]=g(w-\alpha)
$$

4.

$$
F[f(x-\alpha)]=e^{-i \alpha w} g(w)
$$

5.

$$
F\left[e^{i \alpha x}\right]=\delta(w-\alpha)
$$

donde $\alpha$ y $\beta$ son constantes y $\delta(w)$ es la función delta de Dirac.

Como se presenta a continuación, es posible obtener una solución analítica de la EDP de valoración de opciones asiáticas aritméticas continuas mediante cambios de variables y la aplicación de la transformada de Fourier. Un primer cambio de variable es:

$$
V\left(t, S_{t}, A_{t}\right)=e^{r_{f} t} f\left(t, S_{t}, A_{t}\right)
$$

luego: 


$$
\begin{gathered}
\frac{\partial V}{\partial t}=\left(e^{r_{f} t} \frac{\partial f}{\partial t}+r_{f} e^{r_{f} t} f\right) \\
\frac{\partial V}{\partial S_{t}}=e^{r_{f} t} \frac{\partial f}{\partial S_{t}} \\
\frac{\partial^{2} V}{\partial S_{t}^{2}}=e^{r_{f} t} \frac{\partial^{2} f}{\partial S_{t}^{2}} \\
\frac{\partial V}{\partial A_{t}}=e^{r_{f} t} \frac{\partial f}{\partial A_{t}}
\end{gathered}
$$

y la ecuación se transforma en:

$$
\begin{gathered}
\frac{\partial V}{\partial t}+\frac{\sigma^{2} S_{t}^{2}}{2} \frac{\partial^{2} V}{\partial S_{t}^{2}}+r_{f} S_{t} \frac{\partial V}{\partial S_{t}}+S_{t} \frac{\partial V}{\partial A_{t}}-r_{f} V=0 \\
\left(e^{r_{f} t} \frac{\partial f}{\partial t}+r_{f} e^{r_{f} t} f\right)+\frac{\sigma^{2} S_{t}^{2}}{2} e^{r_{f} t} \frac{\partial^{2} f}{\partial S_{t}^{2}}+r_{f} S_{t} e^{r_{f} t} \frac{\partial f}{\partial S_{t}}+S_{t} e^{r_{f} t} \frac{\partial f}{\partial A_{t}}-r_{f} e^{r_{f} t} f=0 \\
\frac{\partial f}{\partial t}+\frac{\sigma^{2} S_{t}^{2}}{2} \frac{\partial^{2} f}{\partial S_{t}^{2}}+r_{f} S_{t} \frac{\partial f}{\partial S_{t}}+S_{t} \frac{\partial f}{\partial A_{t}}=0
\end{gathered}
$$

considerando ahora el cambio de variable $Z_{t}=\ln \left(S_{t}\right)$ con $S_{t}>0$, se tiene que $f\left(t, Z_{t}, A_{t}\right)$ de donde:

$$
\frac{\partial f}{\partial S_{t}}=\frac{\partial f}{\partial Z_{t}} \frac{\partial Z_{t}}{\partial S_{t}}=\frac{\partial f}{\partial Z_{t}} \frac{1}{S_{t}}, \quad \text { luego } \quad S_{t} \frac{\partial f}{\partial S_{t}}=\frac{\partial f}{\partial Z_{t}}
$$

$\mathrm{y}$

$$
\frac{\partial^{2} f}{\partial S_{t}^{2}}=\frac{\partial f}{\partial Z_{t}}\left(-\frac{1}{S_{t}^{2}}\right)+\frac{1}{S_{t}}\left(\frac{\partial^{2} f}{\partial Z_{t}^{2}} \frac{1}{S_{t}}\right), \quad \text { luego } \quad S_{t}^{2} \frac{\partial^{2} f}{\partial S_{t}^{2}}=\frac{\partial^{2} f}{\partial Z_{t}^{2}}-\frac{\partial f}{\partial Z_{t}}
$$

con lo cual la ecuación (15) se transforma en:

$$
\frac{\partial f}{\partial t}+\frac{\sigma^{2}}{2}\left(\frac{\partial^{2} f}{\partial Z_{t}^{2}}-\frac{\partial f}{\partial Z_{t}}\right)+r_{f} \frac{\partial f}{\partial Z_{t}}+e^{Z_{t}} \frac{\partial f}{\partial A_{t}}=0
$$

Con el cambio de variable $\tau=T-t$, luego $\frac{\partial f}{\partial \tau}=-\frac{\partial f}{\partial t}$, entonces: 


$$
-\frac{\partial f}{\partial \tau}+\frac{\sigma^{2}}{2} \frac{\partial^{2} f}{\partial Z_{t}^{2}}+\left(r_{f}-\frac{\sigma^{2}}{2}\right) \frac{\partial f}{\partial Z_{t}}+e^{Z_{t}} \frac{\partial f}{\partial A_{t}}=0
$$

y asumiendo que $Z_{t}=-i Y_{t}$, donde $i=\sqrt{-1}$ es la unidad imaginaria, se tiene que:

$$
\frac{\partial f}{\partial Z_{t}}=-i \frac{\partial f}{\partial Y_{t}} \quad ; \quad \frac{\partial^{2} f}{\partial Z_{t}^{2}}=(-i)^{2} \frac{\partial^{2} f}{\partial Y_{t}^{2}}=-\frac{\partial^{2} f}{\partial Y_{t}^{2}}
$$

luego,

$$
-\frac{\partial f}{\partial \tau}-\frac{\sigma^{2}}{2} \frac{\partial^{2} f}{\partial Y_{t}^{2}}-i\left(r_{f}-\frac{\sigma^{2}}{2}\right) \frac{\partial f}{\partial Y_{t}}+e^{-i Y_{t}} \frac{\partial f}{\partial A_{t}}=0
$$

La ecuación (18) es de coeficientes constantes, excepto en el término asociado con $\frac{\partial f}{\partial A_{t}}$; aplicando la transformada de Fourier a cada término de la ecuación, partiendo de la función $f\left(\tau, Y_{t}, A_{t}\right)$ y llegando a la función $g\left(\tau, w, A_{t}\right)$, se tiene:

$$
F\left[-\frac{\partial f}{\partial \tau}\right]=-\frac{\partial g\left(\tau, w, A_{t}\right)}{\partial \tau}
$$

$$
\begin{aligned}
F\left[-\frac{\sigma^{2}}{2} \frac{\partial^{2} f}{\partial Y_{t}^{2}}\right] & =\frac{-\sigma^{2}}{2} F\left[\frac{\partial^{2} f}{\partial Y_{t}^{2}}\right]=\frac{-\sigma^{2}}{2}(i w)^{2} g\left(\tau, w, A_{t}\right) \\
& =\frac{\sigma^{2} w^{2}}{2} g\left(\tau, w, A_{t}\right)
\end{aligned}
$$

$$
\begin{aligned}
F\left[-i\left(r_{f}-\frac{\sigma^{2}}{2}\right) \frac{\partial f}{\partial Y_{t}}\right] & =-i\left(r_{f}-\frac{\sigma^{2}}{2}\right) F\left[\frac{\partial f}{\partial Y_{t}}\right] \\
& =-i(i w)\left(r_{f}-\frac{\sigma^{2}}{2}\right) g\left(\tau, w, A_{t}\right) \\
& =w\left(r_{f}-\frac{\sigma^{2}}{2}\right) g\left(\tau, w, A_{t}\right)
\end{aligned}
$$

$$
F\left[e^{-i Y_{t}} \frac{\partial f}{\partial A_{t}}\right]=\frac{\partial g\left(\tau, w+1, A_{t}\right)}{\partial A_{t}}
$$


y la ecuación (18) se transforma en:

$$
\begin{gathered}
-\frac{\partial g\left(\tau, w, A_{t}\right)}{\partial \tau}+\frac{\sigma^{2} w^{2}}{2} g\left(\tau, w, A_{t}\right)+w\left(r_{f}-\frac{\sigma^{2}}{2}\right) g\left(\tau, w, A_{t}\right)+\frac{\partial g\left(\tau, w+1, A_{t}\right)}{\partial A_{t}}=0 \\
\frac{\partial g\left(\tau, w, A_{t}\right)}{\partial \tau}=\left[\frac{\sigma^{2} w^{2}}{2}+w\left(r_{f}-\frac{\sigma^{2}}{2}\right)\right] g\left(\tau, w, A_{t}\right)+\frac{\partial g\left(\tau, w+1, A_{t}\right)}{\partial A_{t}}
\end{gathered}
$$

Asumiendo que:

$$
g\left(\tau, w, A_{t}\right)=\exp \left\{\left[\frac{\sigma^{2} w^{2}}{2}+w\left(r_{f}-\frac{\sigma^{2}}{2}\right)\right] \tau\right\} h\left(\tau, w, A_{t}\right)
$$

la ecuación (20) se transforma en:

$$
\frac{\partial h\left(\tau, w, A_{t}\right)}{\partial \tau}=\frac{\partial h\left(\tau, w+1, A_{t}\right)}{\partial A_{t}}
$$

y aplicando nuevamente la transformada de Fourier sobre la ecuación (22) en la variable $w$ se tiene que:

$$
\frac{\partial \bar{h}\left(\tau, \bar{w}, A_{t}\right)}{\partial \tau}=\frac{\partial \bar{h}\left(\tau, \bar{w}, A_{t}\right)}{\partial A_{t}} e^{i \bar{w}}
$$

donde $F\left[h\left(\tau, w, A_{t}\right)\right]=\bar{h}\left(\tau, \bar{w}, A_{t}\right)$. Una solución de esta ecuación es:

$$
\bar{h}\left(\tau, \bar{w}, A_{t}\right)=c\left(\tau+A_{t} e^{-i \bar{w}}\right)
$$

donde $c$ es una constante, y se tiene la condición de frontera:

$$
\bar{h}\left(0, \bar{w}, A_{t}\right)=\phi\left(\bar{w}, A_{t}\right)=c\left(A_{t} e^{-i \bar{w}}\right)
$$

de donde,

$$
\begin{gathered}
c=\frac{\phi\left(\bar{w}, A_{t}\right)}{A_{t} e^{-i \bar{w}}}=\bar{\phi}\left(\bar{w}, A_{t}\right) \\
\bar{h}\left(\tau, \bar{w}, A_{t}\right)=\bar{\phi}\left(\bar{w}, A_{t}\right)\left(\tau+A_{t} e^{-i \bar{w}}\right)
\end{gathered}
$$

Aplicando la transformada inversa de Fourier en $\bar{w}$ se tiene que:

$$
h\left(\tau, w, A_{t}\right)=\phi\left(w, A_{t}\right)\left[\tau \delta(w)+A_{t} \delta(w-1)\right]
$$


luego,

$g\left(\tau, w, A_{t}\right)=\exp \left\{\left[\frac{\sigma^{2} w^{2}}{2}+w\left(r_{f}-\frac{\sigma^{2}}{2}\right)\right] \tau\right\} \phi\left(w, A_{t}\right)\left[\tau \delta(w)+A_{t} \delta(w-1)\right]$

$\mathrm{y}$

$$
f\left(\tau, Y_{t}, A_{t}\right)=F^{-1}\left[g\left(\tau, w, A_{t}\right)\right]
$$

Dado que $Y_{t}=-i Z_{t}, S_{t}=e^{Z_{t}}$ y $V=e^{r_{f} t} f$, se tiene entonces que:

$$
\begin{aligned}
& f\left(\tau, Y_{t}, A_{t}\right)=\frac{1}{2 \pi} \int_{-\infty}^{\infty} \exp \left\{\left[\frac{\sigma^{2} w^{2}}{2}+w\left(r_{f}-\frac{\sigma^{2}}{2}\right)\right] \tau\right\} \phi\left(w, A_{t}\right)\left[\tau \delta(w)+A_{t} \delta(w-1)\right] e^{i w y} d w \\
& f\left(\tau, Z_{t}, A_{t}\right)=\frac{1}{2 \pi} \int_{-\infty}^{\infty} \exp \left\{\left[\frac{\sigma^{2} w^{2}}{2}+w\left(r_{f}-\frac{\sigma^{2}}{2}\right)\right] \tau\right\} \phi\left(w, A_{t}\right)\left[\tau \delta(w)+A_{t} \delta(w-1)\right] e^{w z} d w \\
& f\left(t, S_{t}, A_{t}\right)=\frac{1}{2 \pi} \int_{-\infty}^{\infty} \exp \left\{\left[\frac{\sigma^{2} w^{2}}{2}+w\left(r_{f}-\frac{\sigma^{2}}{2}\right)\right](T-t)\right\} S_{t}^{w} \phi\left(w, A_{t}\right)\left[\tau \delta(w)+A_{t} \delta(w-1)\right] d w
\end{aligned}
$$

Finalmente, se llega a la expresión para el valor de la opción:

$$
V\left(t, S_{t}, A_{t}\right)=\frac{1}{2 \pi} \int_{-\infty}^{\infty} e^{\left[\frac{\sigma^{2} w^{2}}{2}+w\left(r_{f}-\frac{\sigma^{2}}{2}\right)-r_{f}\right](T-t)} S_{t}^{w} \phi\left(w, A_{t}\right)\left[\tau \delta(w)+A_{t} \delta(w-1)\right] d w
$$

\section{Conclusiones y futura investigación}

La valoración de opciones asiáticas aritméticas continuas ha sido un desafío recurrente en el campo de finanzas cuantitativas, dada la dificultad para encontrar expresiones analíticas para la distribución de la integral de variables lognormales. En este artículo se establece una ecuación diferencial parcial de segundo orden asociada al problema de valoración de este tipo de opciones, y se presenta una aproximación a su solución mediante la aplicación de la transformada de Fourier a los términos de la ecuación que resulta después de una serie de cambios de variable. La aplicación de la transformada resulta en una ecuación diferencial parcial de primer orden con coeficientes constantes y de fácil resolución. 
Sobre la solución de la ecuación diferencial parcial de primer orden se aplica la transformada inversa para llegar a una expresión analítica. El resultado encontrado se puede extender fácilmente al caso en el cual el activo subyacente paga dividendos compuestos continuamente a una tasa $y$. Como futura investigación se propone considerar la combinación del método de transformada de Fourier con otro tipo de transformación integral como la de Laplace o Mellin, que han demostrado ser eficientes en problemas de valoración de opciones exóticas. 


\section{Referencias}

Barucci, E., Polidoro, S., y Vespri, V. (2001). Some results on partial differential equations and asian options. Mathematical Models and Methods in Applied Sciences, 11(03), 475-497.

Dubois, F., y Lelièvre, T. (2004). Efficient pricing of asian options by the pde approach. Journal of Computational Finance, 8(2), 55-64.

Rogers, L. C. G., y Shi, Z. (1995). The value of an asian option. Journal of Applied Probability, 32(4), 1077-1088.

Vecer, J. (2001). A new pde approach for pricing arithmetic average asian options. Journal of computational finance, 4(4), 105-113.

Yor, M. (2001). Bessel processes, asian options, and perpetuities. En Exponential functionals of brownian motion and related processes (pp. 63-92). New York: Springer.

Zhang, J. E. (2003). Pricing continuously sampled asian options with perturbation method. Journal of Futures Markets: Futures, Options, and Other Derivative Products, 23(6), 535-560. 